\title{
Evaluación de accesibilidad web de las universidades chilenas
}

\author{
Carlos Máñez Carvajal \\ Universidad Católica de Valencia San Vicente Mártir, c/ Sagrado Corazón, 5. 46110 Godella-España \\ (correo-e: carlos.manez@ucv.es)
}

Recibido Dic. 23, 2019; Aceptado Feb. 25, 2020; Versión final Abr. 27, 2020, Publicado Oct. 2020

\begin{abstract}
Resumen
El presente trabajo muestra los resultados de un estudio de accesibilidad web de instituciones universitarias en Chile. El análisis ha sido realizado en base a las pautas de accesibilidad para el contenido web (WCAG 2.0), estándar internacional establecido por el World Wide Web Consortium. Para llevarlo a cabo se han utilizado diferentes herramientas automáticas de evaluación de la accesibilidad web y se ha tomado como referencia la página inicial de cada estamento universitario. El estudio revela que los sitios web de las universidades chilenas presentan barreras de acceso a la información para las personas con discapacidad, mostrando un escaso cumplimiento de la normativa. Se concluye que los problemas detectados en las instituciones residen principalmente en la codificación HTML implementada en las páginas web, en la percepción de la información y en la ausencia de mecanismos de ayuda al usuario mientras interactúa con el contenido web.
\end{abstract}

Palabras clave: accesibilidad web; universidades chilenas; plataformas universitarias; WCAG 2.0

\section{Web accessibility evaluation of Chilean universities}

\section{Abstract}

This research article examined the web accessibility of Chilean universities. The analysis was conducted based on the Web Content Accessibility Guidelines (WCAG) 2.0, an international standard established by the World Wide Web Consortium. The analysis was performed using automated web accessibility assessment tools and the home page of each university. The results revealed that Chilean universities websites presented barriers for information accessing to people with disabilities. These websites showed scarce compliance with standard guidelines. It is concluded that the problems detected in the examined universities were mainly due to the HTML encoding implemented on their websites, information perception, and the lack of user assistant mechanisms for web content interaction. 


\section{INTRODUCCIÓN}

Vivimos en la era de las Tecnologías de la Información y la Comunicación (TIC) en la cual se ha constituido y acrecentado un modelo de Universidad Digital (Laviña y Mengual, 2008). Se establece una modalidad de enseñanza virtual donde las universidades ofrecen nuevas oportunidades y posibilidades a personas que antes tenían dificultades en acceder a una formación superior. Esta modalidad de enseñanza resulta muy acorde con una de las misiones de la Organización de las Naciones Unidas para la Educación, la Ciencia y la Cultura (UNESCO), proporcionar una educación de calidad para todos y el aprendizaje a lo largo de toda la vida. Prácticamente todas las universidades ofrecen espacios virtuales para desarrollar el proceso enseñanza y aprendizaje apoyado por el continuo y constante desarrollo de las nuevas tecnologías.

La Universidad Digital se ha visto extraordinariamente favorecida en los últimos años por la expansión de Internet y la proliferación de dispositivos tecnológicos de última generación, resultando casi extraño hoy en día realizar un curso sin un componente virtual. Sin embargo, la emergencia del aprendizaje en línea puede acarrear desafíos y barreras para las personas si los sitios web universitarios no tienen en cuenta las necesidades y requisitos de los individuos.

Las personas mayores y las personas con discapacidad pueden interactuar con el contenido web utilizando distintos medios y tecnologías como teclados o ratones alternativos o lectores de pantalla denominados productos de apoyo. Por este motivo, resulta fundamental que las páginas web se implementen utilizando las mejores prácticas en diseño y accesibilidad, para facilitar el acceso a la información. Las instituciones de educación superior tienen la obligación de conseguir una igualdad de acceso a las oportunidades educativas, así como asegurar que los estudiantes en situación de discapacidad tengan la posibilidad de acceder a todos sus recursos, sin ningún tipo de discriminación (Fernández-Morales y Duarte, 2016). Sin embargo, la mayoría de sitios web presentan barreras que dificultan y en ocasiones imposibilitan a algunas personas el poder usarlos (Harper y Chen, 2012). Brajnik (2008) describe estas barreras electrónicas como cualquier condición que obstaculiza el progreso de un usuario cuando navega por una web y utiliza productos de apoyo.

El estamento que vela por un adecuado desarrollo de la web es el World Wide Web Consortium (W3C) que define la accesibilidad web como un acceso universal a la Web, independientemente del tipo de hardware, software, infraestructura de red, idioma, cultura, localización geográfica y capacidades de los usuarios (W3C, 2008). Con la finalidad de preservar los derechos de todas las personas, el W3C creó la Web Accesibility Initiative (WAI), grupo de trabajo encargado de establecer las pautas de accesibilidad al contenido web, Web Content Accesibility Guidelines (WCAG) para guiar el diseño web hacia un diseño universal, tratando que los contenidos sean accesibles para un mayor número de personas. Estas pautas han evolucionado desde su creación (WCAG 1.0) hasta la última versión publicada por el W3C, las WCAG 2.1.

Las WCAG 2.0 se basan en cuatro principios fundamentales que se exponen a continuación:1) Perceptible (la información y los componentes de la interfaz de usuario de la web, deben ser presentados a los usuarios de modo que ellos puedan percibirlos); 2) Operable (los componentes web que forman parte de la interfaz y la navegación deben poder ser manejados por usuarios con distintas habilidades y diferentes capacidades); 3) Comprensible (la información presentada como el manejo de la interfaz de usuario debe ser fácilmente comprensible; y 4) Robusto (el contenido debe ser lo suficientemente robusto para que pueda ser interpretado por una amplia variedad de agentes de usuario, incluyendo los productos de apoyo). Cada principio está formado por un número variable de pautas que contienen una serie de puntos de verificación a los que se les asigna un nivel de conformidad que un desarrollador web debe cumplir para lograr que el contenido web sea más accesible para cualquier usuario.

Los niveles de conformidad son los siguientes:1) A (es el requerimiento mínimo de accesibilidad y caso de no cumplirse, impediría el acceso a un gran número de usuarios); 2) AA (debe ser cumplido, pues de lo contrario ciertos grupos de usuarios tendrían dificultades de acceso); 3) AAA (el nivel más avanzado en cuanto a accesibilidad web, caso de no cumplirse algunos usuarios podrían experimentar dificultades en el acceso. (W3C, 2018). Están consideradas un estándar internacional en la mayoría de los países y se encuentran reconocidas legislativamente para los sitios web dependientes del estado. En el caso de Chile, la Ley 20.422 contempla que las instituciones de educación superior deben proveer los mecanismos necesarios que faciliten el acceso de las personas con discapacidad a las instituciones, materiales y medios de enseñanza para que puedan cursar estudios universitarios.

Los estudios de accesibilidad web de estamentos de educación superior siempre han despertado el interés de los investigadores. Los organismos evaluados, en la mayoría de los casos difícilmente cumplen con el estándar internacional. La investigación realizada por Kurt (2011) relativa al ámbito universitario turco denotaba que todos los estamentos analizados presentaban errores de accesibilidad web. El autor reanalizó las mismas instituciones cinco años más tarde mostrando que el nivel de accesibilidad web de los estamentos 
había disminuido (Kurt, 2017). Resultados muy severos fueron obtenidos por Laitano (2015) al analizar 20 universidades pertenecientes al espacio público argentino. Las dificultades de acceso fueron consideradas graves o muy graves. Las barreras más frecuentes estaban relacionadas con la sintaxis del lenguaje de marcado, con la presentación del contenido, con el contenido no textual y con la legibilidad visual del texto.

Chacón-Medina et al. (2013) analizaron dos páginas web de 74 universidades españolas, se revisaron principios, pautas y criterios de conformidad de la norma, en sus niveles de conformidad A y AA. El estudio reveló que la totalidad de las instituciones de educación superior analizadas presentaban barreras de acceso a la información. Resultados similares obtuvieron Verkijika y De Wet (2018) al analizar 26 instituciones de educación superior sudafricanas, todas necesitaban realizar ajustes para alcanzar los criterios de las WCAG 2.0. Campoverde-Molina et al. (2019) evaluaron la accesibilidad de 55 portales web de universidades ecuatorianas utilizando herramientas automáticas de evaluación. Ninguna de las 220 páginas web analizadas cumplía con la normativa técnica vigente en Ecuador.

Ismailova y Inal (2017) realizaron un test entre las mejores universidades de Turquía, República Kirguisa, Azerbaiyán y Kazajstán, el resultado del mismo indicaba que la mayoría de ellas no cumplía con la normativa WCAG 2.0. Nir y Rimmerman (2018) examinaron la accesibilidad web en el contexto de la educación superior israelí, todas las páginas web de las instituciones presentaban barreras de accesibilidad y carecían del nivel de conformidad más básico.

Los estudios de accesibilidad web en el espacio web universitario chileno son muy escasos, el más reciente, fue elaborado por Máñez-Carvajal et al. (2018) quienes analizaron las universidades chilenas presentes en el ranking The World University Rankings. Los resultados del estudio mostraron carencias y dificultades de acceso para las personas con discapacidad en las 13 instituciones analizadas. La revisión de la literatura permite comprobar que la mayoría de las instituciones universitarias analizadas tenían o siguen teniendo dificultades en el cumplimiento de la normativa WCAG 2.0.

\section{MATERIALES Y MÉTODOS}

El objetivo principal de esta investigación ha sido analizar la accesibilidad web de las instituciones universitarias de Chile en base a las WCAG 2.0. Se tomó como referencia las 57 universidades que conforman la educación superior de Chile. Para llevar a cabo este tipo de estudios, es posible utilizar herramientas software que ayudan a evaluar el nivel de accesibilidad de un sitio web (Serrano et al., 2010). Estas herramientas analizan el código fuente y señalan el lugar exacto donde se encuentra el error, pero no pueden determinar si una página web es o no accesible, para ello resulta necesaria una comprobación humana (W3C, 2019). Reducen significativamente el tiempo y esfuerzo que requiere llevar a cabo un análisis de accesibilidad de una página web y son especialmente útiles a la hora de realizar una valoración preliminar.

Se seleccionaron las páginas a evaluar de cada institución y las herramientas automáticas de evaluación de la accesibilidad web para realizar el estudio en base a las WCAG 2.0. El resultado de la evaluación de las herramientas automáticas fue registrado y almacenado en documentos Excel. Posteriormente, se analizaron los resultados utilizando el programa Statistical Package for the Social Sciences (SPSS). La investigación fue llevada a cabo en mayo de 2019 y los datos obtenidos se depositaron en Mendeley Data, un repositorio de acceso público.

\section{Selección de las páginas a evaluar}

Se seleccionó la página inicial de cada estamento pues es el documento más representativo y de mayor importancia de cualquier organismo. Se trata de un elemento común que contiene enlaces vinculantes a los espacios web más importantes de cada institución y en caso de que no resulte accesible para un usuario, difícilmente podrá acceder a otras páginas web.

\section{Selección de las herramientas automáticas de evaluación}

En primer lugar, se testeó la gramática HTML empleada en las páginas web para determinar si permite una adecuada interacción con los diferentes productos de apoyo que puede utilizar una persona con discapacidad para acceder a los contenidos web. Se utilizó W3C Markup Validation Service (W3C, 2019) que permite verificar si una web contiene errores en su código fuente. Para realizar el estudio de accesibilidad web se empleó el Test de accesibilidad Web (TAW), esta aplicación realiza un análisis de la página web en función de las WCAG 2.0 y proporciona un informe detallado con los errores y advertencias de cada página, organizando toda la información en base a los cuatro principios fundamentales de accesibilidad web (Perceptible, Operable, Comprensible y Robusto). Se especificó el nivel de conformidad AA en la evaluación de las páginas web. 


\section{RESULTADOS Y DISCUSIÓN}

EI análisis realizado por W3C Markup Validation Service muestra que la totalidad de las instituciones presentan errores en la codificación HTML de las páginas web. Los resultados de la evaluación con TAW revelan carencias en el cumplimiento de la normativa y la existencia de posibles barreras de acceso en el caso de que una persona con discapacidad visite la web.

La Tabla 1 muestra el número total de errores y la frecuencia de error en los puntos de verificación de la población analizada. Todos los puntos de verificación tienen asociado un nivel de conformidad $A$, el requisito mínimo de accesibilidad. El punto de verificación 2.4.4 propósito de los enlaces es el que presenta mayor número de errores $(n=1395)$ y se repite en el $95.5 \%$ de las páginas web de las instituciones. El segundo punto de verificación que precisa más mejoras es 4.1.1 Procesamiento $(n=1321)$ y se repite en el $94.7 \%$ de las webs analizadas. La Figura 1 muestra los mismos resultados obtenidos por TAW agrupados por pauta. Las pautas Compatible, Navegable y Alternativas textuales son las que presentan el número más elevado de errores de accesibilidad web y por tanto, las que requieren mayor atención por parte de los desarrolladores web. Tras categorizar los resultados del análisis por principio (Figura 2) se observa que Robusto, Operable y Perceptible son los principios con mayor número de errores.

Tabla 1: Número y frecuencia de error en los puntos de verificación de la población analizada

\begin{tabular}{|l|l|l|}
\hline Punto de verificación & № total de errores & Frecuencia (\%) \\
\hline 2.4.4 Propósito de los enlaces & 1395 & 95.5 \\
\hline 4.1.1 Procesamiento & 1321 & 94.7 \\
\hline 1.1.1 Contenido no textual & 908 & 93 \\
\hline 1.3.1 Información y relaciones & 381 & 96.5 \\
\hline 4.1.2 Nombre, función, valor (interfaz usuario) & 170 & 84.2 \\
\hline 3.3.2 Etiquetas o instrucciones & 115 & 68.4 \\
\hline 3.2.2 Introducir datos & 34 & 42.1 \\
\hline 3.1.1 Idioma de la página & 12 & 21.1 \\
\hline 2.2.2 Pausar, detener, ocultar & 1 & 1.8 \\
\hline 2.4.2 Páginas tituladas & 1 & 1.8 \\
\hline
\end{tabular}

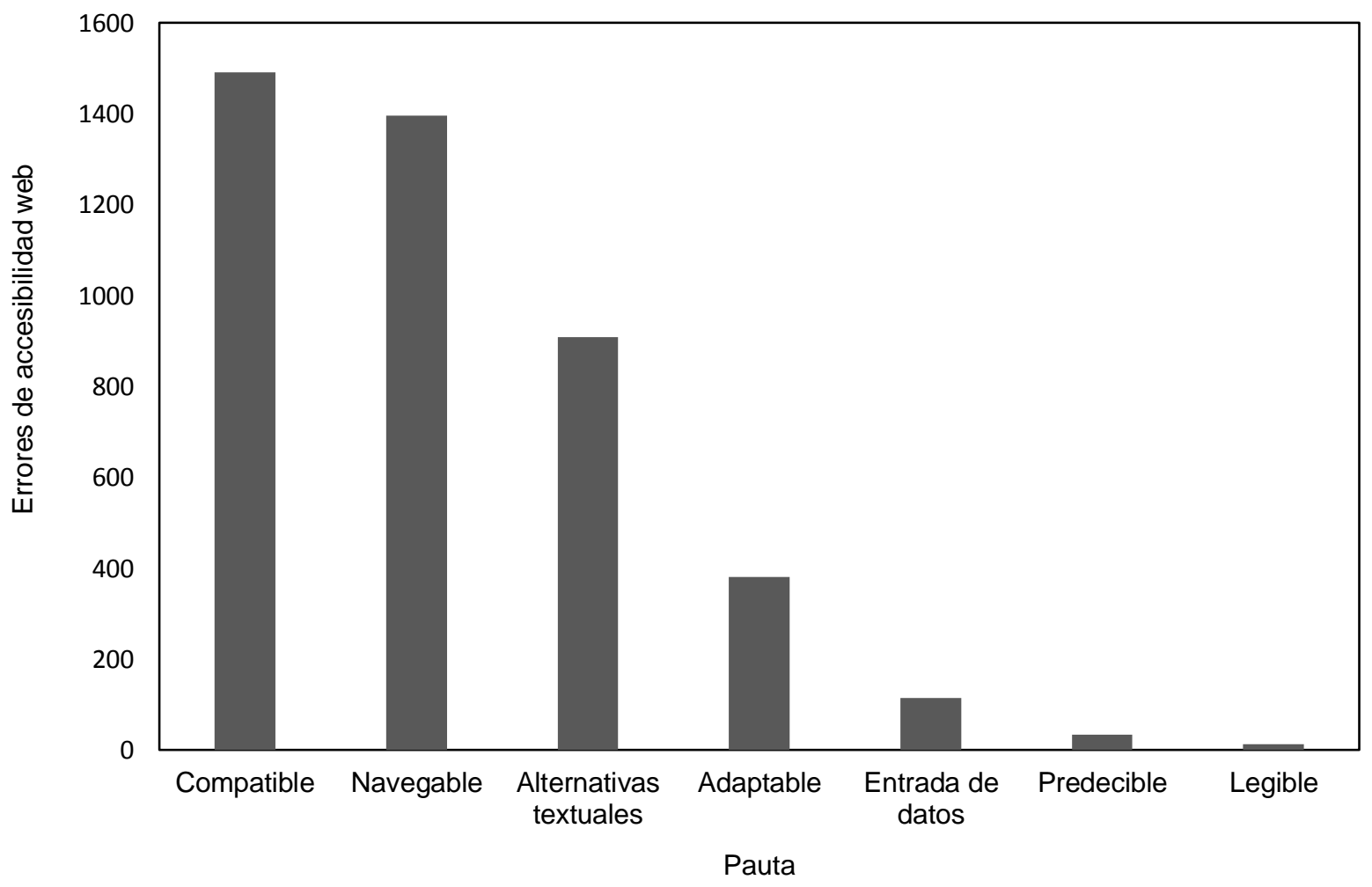

Fig. 1: Errores de accesibilidad web agrupados por Pauta 


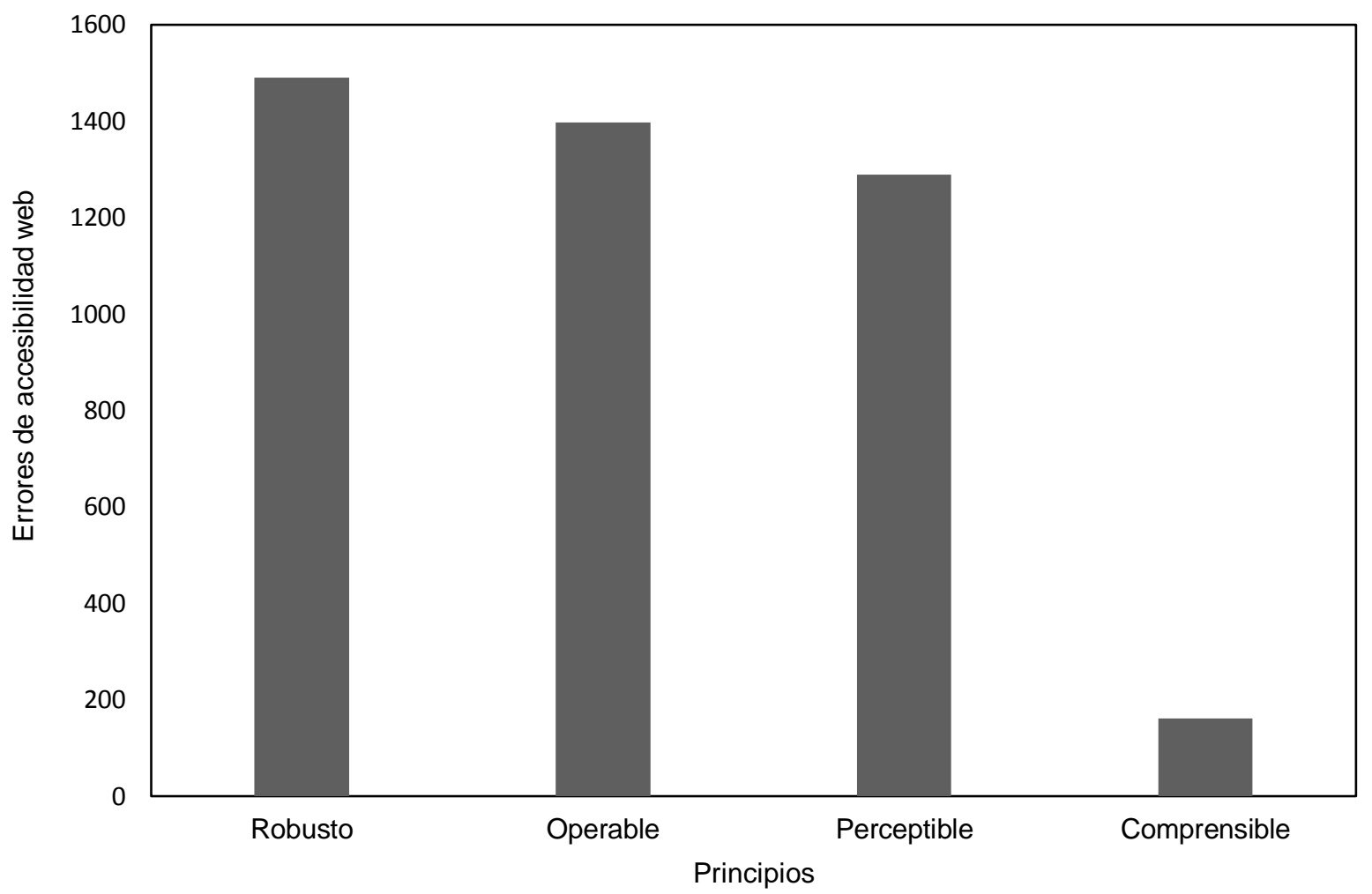

Fig. 2: Errores de accesibilidad web categorizados por principio

\section{Análisis de la distribución}

Se realizó un análisis de los resultados obtenidos por TAW utilizando el programa SPSS. La Tabla 2 muestra los estadísticos descriptivos del conjunto de webs analizadas. Se contabilizó el total de los errores de cada institución en la variable Totales, cuyo rango oscila entre un mínimo de 1 y un máximo de 579, con un promedio de 76.1 errores. Su distribución es claramente asimétrica positiva (asimetría $=3.76$ ) y leptocúrtica (curtosis = 16.24), debido a la acumulación de webs cuyas puntuaciones de sitúan en el extremo inferior de la escala y escasez de webs con puntuaciones en el extremo superior de la escala. En este tipo de distribuciones se considera que la mejor medida de tendencia central no es la media, sino la mediana (Pardo et al., 2009), que tiene un valor de 52 errores. Este valor central de la distribución debe considerarse alto, por lo que se puede afirmar que la cantidad de errores totales es elevada en el conjunto de las webs analizadas. Se puede observar la distribución en el histograma de frecuencias de la Figura 3. En el diagrama de cajas y bigotes (Figura 4) se aprecia claramente la presencia de dos elementos atípicos y dos elementos extraordinariamente atípicos. Estos elementos atípicos junto con la acumulación de datos entorno a la mediana condicionan el carácter asimétrico y leptocúrtico de la distribución.

Tabla 2: Estadísticos descriptivos del conjunto de webs analizadas

\begin{tabular}{|l|l|}
\hline Media & 76.1 \\
\hline Error estándar de la media & 12.84 \\
\hline Mediana & 52 \\
\hline Moda & 18 \\
\hline Desviación estándar & 96.99 \\
\hline Asimetría & 3.76 \\
\hline Error estándar de asimetría & 0.316 \\
\hline Curtosis & 16.241 \\
\hline Error estándar de curtosis & 0.623 \\
\hline Mínimo & 1 \\
\hline Máximo & 579 \\
\hline
\end{tabular}




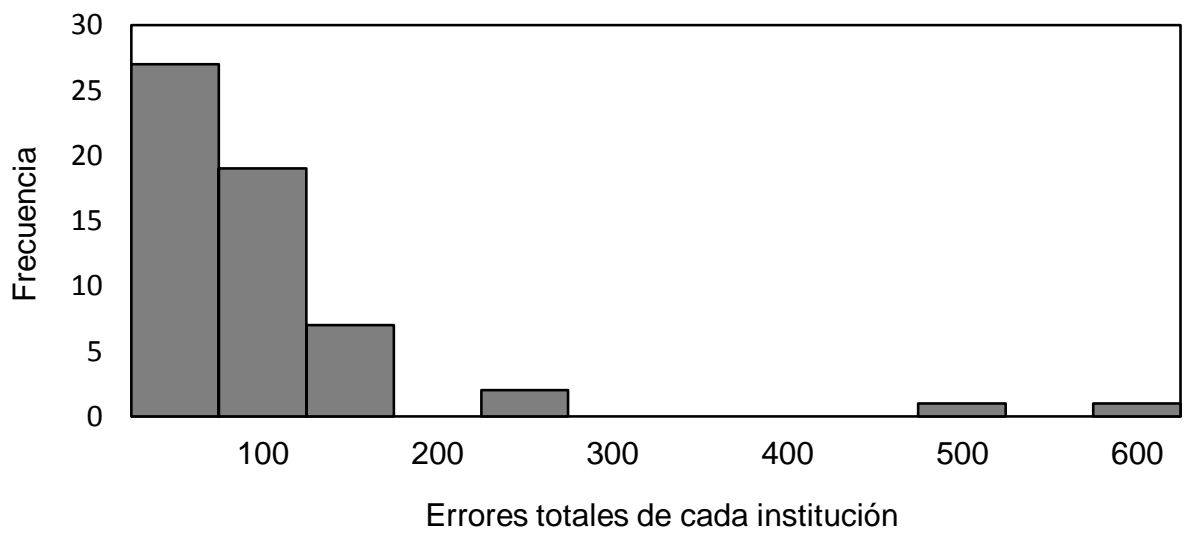

Fig. 3: Histograma de frecuencias

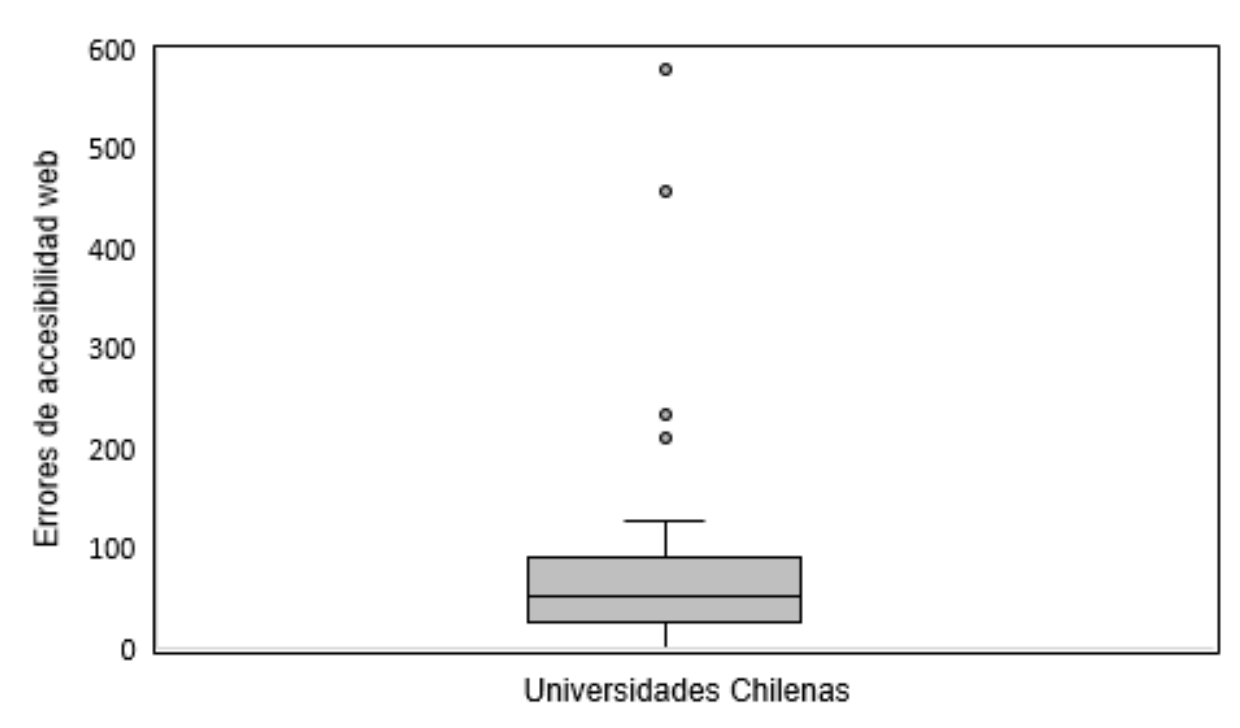

Fig. 4: Diagrama de cajas y bigotes

\section{Análisis y recomendaciones técnicas}

El validador W3C Markup Validation Service encontró errores en la codificación HTML en las páginas web de las instituciones analizadas. Estos resultados aparentemente llamativos no sorprenden demasiado tras la revisión de trabajos anteriores que mostraban que la mayoría de los sitios web analizados presentaban un código fuente con errores (Al-Khalifa, 2012; Basdekis et al., 2010). Los gestores de contenidos utilizados por las instituciones pueden generar o aumentar el número de errores detectados en el lenguaje HTML. Sin embargo, utilizar el W3C Markup Validation Service y eliminar errores no forzados en el código fuente, lograría una mejor interacción con los productos de apoyo que utilizan las personas con discapacidad para navegar por Internet. El análisis realizado por la herramienta TAW muestra que la tipología de los errores detectados por las herramientas de evaluación resulta coincidente con investigaciones actuales de accesibilidad web universitaria (Ismail y Kuppusamy, 2018; Karaim y Inal, 2019; Roig-Vila et al., 2014).

Con respecto al principio Perceptible, observamos que las pautas 1.1 Alternativas textuales y 1.3 Adaptable son las que precisan mayor atención por parte de los desarrolladores. TAW detecta la omisión de alternativas textuales a los contenidos no textuales, principalmente imágenes (1.1.1 Contenido no textual). La ausencia de textos alternativos en imágenes resulta muy común en los estudios de accesibilidad web siendo uno de los errores más fáciles de solucionar. Las descripciones alternativas son elementos básicos que facilitan la percepción de la información a todos y cada uno de los usuarios de la web, pero que resultan fundamentales para una persona que interactúa con la web mediante un lector de pantalla. Además, la herramienta encuentra errores en la adaptación de contenido de acuerdo a las necesidades de cada usuario (1.3.1 Información y relaciones), aspecto especialmente importante si se trata de una persona con discapacidad que utiliza productos de apoyo. Para reducir el número de errores de accesibilidad web, el código fuente de las páginas web debería incluir etiquetas <title $>$ y <alt $>$ y ofrecer alternativas textuales al contenido que lo requiera. Evitar enlaces consecutivos de texto e imágenes al mismo recurso y usar adecuadamente los encabezados en la estructura de las páginas web conllevaría un acercamiento al cumplimiento del estándar internacional. 
En referencia al principio Operable, observamos que la pauta 2.4 Navegable es la que presenta un número más elevado de incidencias, concretamente en el punto de verificación 2.4.4 Propósito de los enlaces. El error que se repite con mayor frecuencia es la omisión de contenido textual en los enlaces, lo que permite al usuario conocer donde se encuentra y hacia dónde va a navegar. Por ello, se deberían implementar mejoras en el código fuente proporcionando contenido textual a los enlaces. Con respecto al principio Comprensible y a la pauta 3.3 Introducción de datos asistida, el tipo de error más común reside en el etiquetado de formularios. Esta pauta resulta de gran ayuda a personas con discapacidad visual que interactúan con la web a través de un lector de pantalla, por tanto, las mejoras en el código fuente deberían estar focalizadas en la implantación de formularios accesibles y un uso adecuado de las etiquetas que los componen (3.3.2 Etiquetas o instrucciones). Otro error común es la omisión de la declaración del idioma del documento HTML que podría ser eliminado fácilmente utilizando la etiqueta <lang $>$.

Los errores detectados en el principio Robusto (4.1.1 Procesamiento) afectan a la estructura y codificación de las páginas web y puede entorpecer o dificultar la experiencia del usuario en el caso de utilice productos de apoyo. Evitar errores en la sintaxis de elementos y atributos, la duplicidad de atributos y hacer un uso adecuado de las etiquetas reduciría las dificultades de acceso de los usuarios. Los análisis proporcionados por las herramientas empleadas en el estudio muestran que las páginas web de las instituciones analizadas carecen de un diseño web que proporcione un acceso equitativo a la información, presentando barreras y dificultades de acceso para las personas mayores y/o con discapacidad.

Estos resultados presentan similitudes con el trabajo anterior más relacionado, que fue llevado a cabo por Máñez-Carvajal et al. (2018) en el que se analizaron 13 instituciones de educación superior chilenas utilizando distintas herramientas de evaluación de la accesibilidad web. En ambos estudios, se testeó la gramática HTML empleada en las páginas web mediante los validadores de código diseñados por el W3C. Ninguna institución presentó un código fuente libre de errores. Las barreras web detectadas por las herramientas resultan coincidentes, en ambos casos, los usuarios de las páginas web experimentan dificultades para percibir la información e interactuar con el contenido web, especialmente si utilizan productos de apoyo. El análisis de los datos en ambos estudios también permite identificar diferencias, la investigación realizada sobre las 13 instituciones reveló que el principio que requería mayor atención por parte de los desarrolladores era Perceptible, con un $40 \%$ de los errores totales de accesibilidad web. Sin embargo, el estudio llevado a cabo sobre las 57 instituciones universitarias muestra un aumento considerable de errores en los principios Robusto y Operable, siendo los que presentan el mayor número de errores de accesibilidad web.

A la vista de los resultados, los desarrolladores de contenidos de las instituciones no tienen en cuenta las directrices del W3C en materia de accesibilidad a la hora de implementar las páginas web. Por ello es necesaria una mayor formación en las WCAG con objeto de ajustar los sitios web a la normativa actual existente, protegiendo de esta manera los derechos fundamentales de las personas y grupos vulnerables.

Las herramientas automáticas de evaluación de la accesibilidad web proporcionan informes muy detallados que combinados con el juicio humano resultan una excelente combinación para detectar y solucionar posibles errores en el cumplimiento de la normativa. Su uso resulta altamente recomendable en las fases de diseño y en el proceso de desarrollo de las webs, reduciendo los errores en accesibilidad y facilitando el acceso a los contenidos. La problemática del aprendizaje en línea y las personas con discapacidad no es en absoluto una novedad, son numerosos los estudios de accesibilidad que muestran barreras de acceso a la información, por este motivo es necesario un cambio conceptual en el diseño y desarrollo de las webs, éstos deben ser encaminados a ser más accesibles y usables por y para, los usuarios de la web.

\section{CONCLUSIONES}

El principal objetivo de esta investigación ha sido evaluar la accesibilidad web de las instituciones universitarias de Chile. Para llevar a cabo el estudio se emplearon herramientas automáticas de accesibilidad web. El resultado de la investigación muestra similitudes con trabajos anteriores de accesibilidad web focalizados en el ámbito universitario. Según lo esperado, las instituciones tienen serias dificultades en el cumplimiento de la normativa vigente, presentando niveles de accesibilidad web bajos o muy bajos y en algunos casos, muy graves. Las páginas web de las universidades carecen de un diseño web que proporcione un acceso equitativo a la información presentando barreras y dificultades de acceso para las personas mayores y/o con discapacidad. Los usuarios de las webs experimentarán dificultades para percibir la información e interactuar con el contenido web, especialmente si utilizan productos de apoyo.

\section{MATERIAL SUPLEMENTARIO}

Los resultados de la evaluación de cada institución se encuentran depositados en el repositorio Mendeley Data bajo una licencia CC BY 4.0 y pueden consultarse en el siguiente enlace: https://data.mendeley.com/datasets/4rhcbhxthh/1 


\section{REFERENCIAS}

Al-Khalifa, H. S., The accessibility of Saudi Arabia government Web sites: An exploratory study, doi:10.1007/s10209-0100215-7, Universal Access in the Information Society, 11(2), 201-210 (2012)

Basdekis, I., I. Klironomos, I. Metaxas y C. Stephanidis, An overview of web accessibility in Greece: A comparative study 2004-2008, doi: 10.1007/s10209-009-0166-z, Universal Access in the Information Society, 9(2), 185-190 (2010)

Brajnik, G., Beyond Conformance: The Role of Accessibility Evaluation Methods, Web Information Systems Engineering - WISE 2008 Workshops, Springer-Verlag, Vol. 5176, pp. 63-80, Berlin Heidelberg, Alemania (2008)

Campoverde-Molina, M., Luján-Mora, S. y Valverde, L., Análisis de accesibilidad web de las universidades y escuelas politécnicas del Ecuador aplicando la norma NTE INEN ISO/IEC 40500:2012. Revista Ibérica de Sistemas e Tecnologias de Informação, 22, 53-68 (2019)

Chacón-Medina, A., H. Chacón-López, M. D. López-Justicia y C. Fernández-Jiménez, Dificultades en la Accesibilidad Web de las Universidades Españolas de acuerdo a la Norma WCAG 2.0, doi: 10.3989/redc.2013.4.1009, Revista española de Documentación Científica, 36(4), 025 (2013)

Fernández-Morales, F. H. y J. E. Duarte, Retos de la inclusión Académica de Personas con Discapacidad en una Universidad Pública Colombiana, doi:10.4067/S0718-50062016000400011, Formación universitaria, 9(4), 95-104, (2016) Harper, S. y A. Q. Chen, Web accessibility guidelines, doi:10.1007/s11280-011-0130-8 World Wide Web, 15(1), 61-88, (2012)

Ismail, A. y K. S. Kuppusamy, Accessibility of Indian universities homepages: An exploratory study, doi: 10.1016/j.jksuci.2016.06.006, Journal of King Saud University - Computer and Information Sciences, 30(2), 268-278 (2018)

Ismailova, R. y Y. Inal, Accessibility evaluation of top university websites: A comparative study of Kyrgyzstan, Azerbaijan, Kazakhstan and Turkey, doi: 10.1007/s10209-017-0541-0, Universal Access in the Information Society, 1-9 (2017)

Karaim, N. A. y Y. Inal, Usability and accessibility evaluation of Libyan government websites, doi: 10.1007/s10209-0170575-3, Universal Access in the Information Society, 18(1), 207-216 (2019)

Kurt, S.,The accessibility of university web sites: The case of Turkish universities, doi: 10.1007/s10209-010-0190-z, Universal Access in the Information Society, 10(1), 101-110 (2011)

Kurt, S., Accessibility of Turkish university Web sites, doi: 10.1007/s10209-016-0468-x, Universal Access in the Information Society, 16(2), 505-515 (2017)

Laitano, M. I., Accesibilidad web en el espacio universitario público argentino, doi: 10.3989/redc.2015.1.1136, Revista española de Documentación Científica, 38(1), e079 (2015)

Laviña, J. y L. Mengual, Libro blanco de la Universidad digital 2010, Ariel, Fundación Telefónica Madrid, España (2008)

Máñez-Carvajal, C., R. Fernández-Piqueras y J.F. Cervera-Mérida. Evaluation of Web Accessibility of Higher Education Institutions in Chile, doi: 10.5539/ies.v11n12p140, International Education Studies, 11(12), p140 (2018)

Nir, H. L. y A. Rimmerman, Evaluation of Web content accessibility in an Israeli institution of higher education, doi: 10.1007/s10209-018-0615-7, Universal Access in the Information Society, 1-11 (2018)

Pardo, A., M. Á. Ruiz y R. San Martín, Análisis de datos en ciencias sociales y de la salud I, Síntesis, Madrid, España (2009)

Roig-Vila, R., S. Ferrández y I. Ferri-Miralles, Assessment of Web Content Accessibility Levels in Spanish Official Online Education Environments, doi: 10.5539/ies.v7n6p31, International Education Studies, 7(6) (2014)

Serrano, E. S., A. M. Ocaña y I. O. Martos, Métrica para la evaluación de la accesibilidad en Internet: Propuesta y testeo, doi: 10.3989/redc.2010.3.719, Revista española de Documentación Científica, 33(3), 378-396 (2010)

Verkijika, S. F. y L. De Wet., Accessibility of South African university websites, doi: 10.1007/s10209-018-0632-6, Universal Access in the Information Society (2018)

W3C, Web Content Accessibility Guidelines (WCAG) 2.0 (2008)

W3C, The W3C Markup Validation Service (2018)

W3C, Introduction to Web Accessibility | Web Accessibility Initiative (WAI) | W3C (2019) 